\title{
Confiabilidade da declaração de causa básica de mortes infantis em região metropolitana do sudeste do Brasil*
}

\author{
Reliability of the medical certificates of underlying cause of infant deaths in a \\ metropolitan region of southeastern Brazil
}

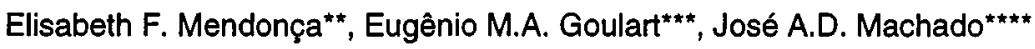

\begin{abstract}
MENDONÇA, E.F. et al. Confiabilidade da declaração de causa básica de mortes infantis em região metropolitana do sudeste do Brasil. Rev. Saúde Pública, 28: 385-91, 1994. A partir de dados coletados para um estudo sobre a mortalidade infantil na região metropolitana de Belo Horizonte, MG, Brasil, foi selecionada uma amostra aleatória de óbitos infantis ocorridos em 1989, para avaliar a concordância da causa básica de morte registrada na declaração de óbito e a obtida após revisão detalhada do prontuário hospitalar da criança. Verificou-se que $11,7 \%$ dos óbitos neonatais não tiveram a causa básica registrada no atestado, confirmada pela investigação nos prontuários médicos (kappa $=0,61)$, o mesmo ocorrendo em 44,0\% dos pós-neonatais (kappa $=0,47$ ). Esta maior discordância no grupo pós-neonatal provavelmente se deveu a maior dificuldade de definição das causas contribuintes e da causa básica dos óbitos por diarréias, pneumonias e desnutrição, principais causas de mortalidade nesse grupo. Em relação aos óbitos por desnutrição e diarréia, observou-se associação entre ambas em $76,9 \%$ das vezes em que a diarréia foi selecionada como causa básica, mostrando que essas patologias podem ser destacadas como um mesmo grupamento em saúde pública. As discordâncias encontradas demonstram que os médicos ainda dão pouca importância ao seu papel como agentes geradores de informação de saúde. Os dados da declaração de óbito fornecem indicação razoável das principais causas de mortes infantis, principalmente quando se considera o grupamento diarréia-pneumonia-desnutrição, composto de patologias evitáveis e ainda de grande relevância como causa de mortalidade infantil na região.
\end{abstract}

Descritores: Causa básica da morte. Mortalidade infantil. Atestados de óbito.

\section{Introdução}

O coeficiente de mortalidade infantil é um dos indicadores mais utilizados para avaliar a situação de saúde da população, considerando que a criança é extremamente sensível às agressões do meio ambiente $^{14}$. No Brasil, este indicador é muitas vezes afetado

*Parte do projeto de pesquisa "Vigilância Epidemiológica e Estudo dos Determinantes da Mortalidade Infantil na Região Metropolitana de Belo Horizonte", financiado pela OPAS (Processo DRC/HPM/RG/BRA/88-099) e FAPEMIG (Fundação de Amparo à Pesquisa do Estado de Minas Gerais). Processo CBS 792-90.

**Departamento de Medicina Preventiva e Social da Faculdade de Medicina da Universidade Federal de Minas Gerais e Escola de Saúde de Minas Gerais/FUNED - Belo Horizonte, MG - Brasil

***Departamento de Pediatria da Faculdade de Medicina da Universidade Federal de Minas Gerais - Belo Horizonte, MG - Brasil

****Departamento de Medicina Preventiva e Social da Faculdade de Medicina da Universidade Federal de Minas Gerais - Belo Horizonte, MG - Brasil

Separatas/Reprints: E.F. Mendonça - Av. Alfredo Balena, 190/

$10^{\circ}$ - 30130-100 - Belo Horizonte, MG - Brasil pelo subregistro de nascimentos e óbitos ${ }^{1,8}$. Na região metropolitana de Belo Horizonte (RMBH) felizmente esta limitação é pequena, pois estima-se que o sub-registro seja pouco significativo ${ }^{17}$. Entretanto, ainda permanece o problema relativo à precisão na declaração da causa básica da morte, de importância fundamental para as estatísticas de mortalidade.

Em 1976, o Ministério da Saúde padronizou um modelo único de declaração de óbito (DO) em nível nacional. Nesse modelo, o médico que preenche a DO é responsável pela determinação da causa básica do óbito, geradora das estatísticas oficiais de mortalidade, e definida como "a doença ou lesão que iniciou a cadeia de acontecimentos patológicos que conduziram diretamente à morte, ou as circunstâncias do acidente ou violência que produziram a lesão fatal"'l3. Esta causa básica deve ser sempre etiologicamente específica e antecedente às outras causas, no tempo e na sequiência patológica. Sem a causa básica, o óbito não deveria ter ocomido ${ }^{7}$.

As causas de morte que devem figurar no atestado de óbito são todas aquelas doenças, estados mórbidos e lesões que produziram a morte ou que contribuíram para ela. Entretanto, a saúde pública tradicionalmente 
só coleta informação quanto à doença inicial da seqüência que levou ao óbito, pois os esforços de prevenção devem se orientar para o início do processo $0^{4}$.

Como muitas vezes o preenchimento da DO não é correto, são utilizadas regras de seleção da causa básica para corrigir sequiências não lógicas e/ou confusas dos eventos que levaram ao óbito. Assim, quando a causa básica é citada fora de sequiência, a mesma é selecionada pela aplicação das chamadas "Regras de Seleção da Causa Básica de Mortalidade", padronizadas internacionalmente e revisadas de $10 \mathrm{em} 10$ anos pela $\mathrm{OMS}^{13}$. Embora as regras guiem o codificador em algumas situações, muitas vezes elas não podem recuperar um atestado preenchido de forma incorreta pelo médico ${ }^{4}$. Quando o preenchimento é correto, a causa básica selecionada para tabulação será a última doença mencionada na parte I da DO.

Vários estudos têm demonstrado a precariedade de preenchimento da DO, levantando muitas desconfianças em relação às estatísticas de mortalidade, principalmente quanto à qualidade da informação sobre a causa do óbito ${ }^{3}$. No Brasil, um dos estudos pioneiros sobre o tema, realizado em São Paulo, em 1962/1963, mostrou que apenas $67,6 \%$ dos óbitos tinham a causa básica registrada em algum campo da $\mathrm{DO}^{10}$. Estudo semelhante foi repetido 10 anos depois, e o percentual de registro da causa básica na DO $(68,6 \%)$ não teve melhoria significativa ${ }^{6}$.

Em relação à mortalidade infantil, em um estudo realizado na região sul do país (Porto Alegre e Pelotas), em 1984-1985, a comparação entre os atestados de óbito oficiais e os revisados pela equipe do estudo mostrou um índice de concordância geral de apenas $27,9 \%$ para os óbitos por doenças infecciosas e desnutrição, ocorridos em crianças entre sete dias e um ano de idade ${ }^{12}$. Outro estudo, realizado na região metropolitana do Rio de Janeiro, em 1986, revelou um índice de concordância de 0,90 (índice kappa de 0,62) para os óbitos neonatais e de 0,61 (kappa de 0,47) para os óbitos pós-neonatais ${ }^{2}$.

O presente estudo procurou investigar a concordância entre a causa básica de morte registrada na DO e a obtida após revisão detalhada do prontuário hospitalar da criança, com o objetivo de avaliar a confiabilidade da DO na definição das principais causas de mortes infantis.

\section{Material e Método}

O presente trabalho foi realizado em Belo Horizonte, capital do Estado de Minas Gerais, e em 13 municípios limítrofes componentes da região metropolitana de Belo Horizonte(RMBH), com uma população total, segundo o último censo brasileiro de 1991, de cerca de 3,4 milhões de habitantes.
Selecionou-se uma amostra aleatória de 195 óbitos infantis hospitalares, dentre 2.830 óbitos de crianças residentes na $\mathrm{RMBH}$, ocorridos em 1989. Para o cálculo do tamanho da amostra, considerou-se uma proporção estimada de discordância na população de $50 \%$, uma precisão de $7 \%$ e um intervalo de confiança de $95 \%$. Para cada declaração de óbito sorteada procedeu-se a uma visita hospitalar, onde foram coletados, em formulário padronizado, dados do prontuário hospitalar. Após análise desses dados, uma equipe de médicos preencheu um novo atestado de óbito (atestado modificado), com especificação das causas da morte, segundo as regras de preenchimento da $\mathrm{DO}^{9}$, sem o conhecimento das informações da DO original (estudo cego). Foram utilizadas pela equipe da pesquisa as mesmas informações normalmente disponíveis aos médicos internistas para certificarem o óbito, com exceção as referentes a 4 óbitos, com resultados posteriores de necrópsia. A qualidade dos registros hospitalares variou muito. Em alguns hospitais, o prontuário era incompleto, dificultando o preenchimento da "nova" DO.

Os atestados preenchidos pelos pesquisadores foram enviados à mesma equipe de codificadores que havia trabalhado na codificação das DO originais, para seleção da causa básica do óbito, segundo as normas padronizadas pela CID $9^{13} \mathrm{e}$ Centro Brasileiro de Classificação de Doenças.

Os 195 óbitos infantis do estudo foram agrupados por faixa etária em neonatais $(0$ a 27 dias completos) e pós-neonatais (após o $28^{\circ}$ dia de vida até um ano incompleto). Foi estudada a concordância geral dentro de cada grupo etário, distribuindo-se os óbitos em 5 grandes grupos: causas perinatais (códigos CID 760 779), anomalias congênitas (740-759), pneumonias (480-486), diarréias (001-009) e desnutrição (260269), além de um sexto grupo com as demais causas (demais códigos). A desnutrição foi considerada como causa básica de óbito somente quando classificada como desnutrição grave, sendo adotado o critério diagnóstico de déficit de $40 \%$ ou mais em relação ao peso esperado para a idade e sexo ${ }^{11}$.

Para cada DO original e investigada pela equipe foram levantadas, além da causa básica, a prematuridade e a desnutrição para análise como causas associadas de óbito.

A distribuição por causas e por idade dos óbitos amostrados não diferiu estatisticamente do universo ( $\mathrm{p}=0,71$ e 0,88 , respectivamente), mostrando que não houve viés na seleção da amostra.

Para analisar a concordância entre a causa básica do atestado médico original e a definida pelos investigadores, utilizou-se, além da concordância observada (calculada pela soma dos valores concordantes observados e dividida pelo número total em estudo), a estatística kappa, que resulta sempre em valores um pouco abaixo da concordância observada, pois 
exclui do resultado a concordância que seria esperada por mero acaso ${ }^{5}$. Utilizou-se o programa Epi Info* para o processamento e análise dos dados e o Epistat** para os cálculos de kappa.

\section{Resultados}

Os 5 grandes grupos de causas especificadas (afecções perinatais, anomalias congênitas, pneumonias, diarréias e desnutrição) foram responsáveis por 91\% dos óbitos ocorridos em 1989. As afecções perinatais foram responsáveis pela maior parte desses óbitos (55\%). Neste grupo, 92 dos 107 óbitos permaneceram com o mesmo diagnóstico do atestado original, o mesmo ocorrendo com 15 dos 19 óbitos do grupo de anomalias congênitas. Passaram a integrar o grupo perinatal 7 óbitos classificados anteriormente em outros grupos. Dos 21 óbitos por diarréia selecionados, apenas 8 mantiveram a causa confirmada. Passaram a compor este grupo 8 óbitos codificados anteriormente em outros grupos. Da mesma forma, dos 20 6bitos codificados como pneumonias, 11 permaneceram no grupo e 6 outros entraram. Em relação à desnutrição, 6 dos 10 óbitos registrados no atestado original como desnutrição permaneceram no grupo e 12 passaram a pertencer a este grupamento. Quanto aos óbitos classificados no grupo de outras causas, 13 permaneceram neste grupamento, passando para o grupo 11, outros (Tabela 1).

Ao se analisar a concordância entre a DO original e a DO "modificada" para os seis grupos de causas, verificou-se para as perinatais uma concordância observada de 0,89 e um índice kappa de 0,77. Este índice foi semelhante para as anomalias congênitas (kappa de 0,72$)$, sendo entretanto bem menor para as diarréias, pneumonias e desnutrição (kappa de 0,37,0,55 e 0,39, respectivamente). Considerando o total dos óbitos, verificou-se uma concordância observada de $0,74 \mathrm{e}$ um índice kappa de 0,62 (Tabela 2).

Ainda na Tabela 2, apresentou-se a relação entre o número de vezes que os grupos de causa aparecem na DO modificada e na DO original. As causas perinatais, as diarréias e as pneumonias foram sobrecitadas no atestado original, quando comparadas à frequiência com que constaram do atestado modificado. Por outro lado, as anomalias congênitas, a desnutrição e outras causas foram subcitadas (por exemplo, para cada diagnóstico de desnutrição no atestado original foram feitos 1,8 diagnósticos pela mesma causa no atestado modificado).

O grupamento de outras causas, por envolver patologias muito diferentes e por sua relativa importância (12,3\% dos óbitos após investigação), foi analisado em separado. Houve predominância de causas infecciosas (meningites - CID 320, bronquite - CID 466, coqueluche - CID 033, sífilis congênita - CID 090), tanto na DO original quanto na DO modificada. Adicionando-se a este grupo as anemias ferroprivas, características de deficiência nutricional, verificou-se que o grupamento doença infecciosa-deficiência nutricional foi responsável por mais da metade dos óbitos classificados neste grupamento.

$\mathrm{Na}$ análise de cada grupo etário separadamente (Tabela3), verificou-se que 11,7\% dos óbitos neonatais e $44,0 \%$ dos pós-neonatais não tiveram a causa básica registrada na $D O$ original, confirmada pela investigação nos prontuários hospitalares. O cálculo do kappa, avaliando a concordância dentro de cada grupo etário, mostrou para o grupo neonatal um kappa de 0,61 e para o pós-neonatal um kappa de 0,47 .

A maior concordância verificada para a mortalidade neonatal deveu-se à predominância das causas perinatais, responsáveis por $81,1 \%$ dos óbitos e que praticamente não mudaram de grupo após a revisão das DO. No entanto, houve alterações significativas em alguns subgrupos de causas perinatais, principalmente o de afeç̧ões maternas, que estavam subestimados no atestado original. Isto ocorreu também para os óbitos inicialmente classificados em categorias mais genéricas como prematuridade e anóxia e hipóxia perinatal (CID 764, 765, 768, 769 e 770) que passaram a ter a causa básica melhor especificada após a investigação (Figura).

Tabela 1 - Número de óbitos infantis, segundo causas básicas de óbito no atestado original e no atestado modificado, região metropolitana de Belo Horizonte, 1989.

\begin{tabular}{|c|c|c|c|c|c|c|c|}
\hline \multirow[b]{2}{*}{$\begin{array}{l}\text { Atestado } \\
\text { original }\end{array}$} & \multicolumn{7}{|c|}{ Atestado modificado } \\
\hline & $\begin{array}{l}\text { Peri- } \\
\text { natais }\end{array}$ & $\begin{array}{l}\text { Congê- } \\
\text { nitas }\end{array}$ & $\begin{array}{l}\text { Diar- } \\
\text { réia }\end{array}$ & $\begin{array}{l}\text { Pneu- } \\
\text { monia }\end{array}$ & $\begin{array}{l}\text { Desnu- } \\
\text { trição }\end{array}$ & $\begin{array}{l}\text { Outras } \\
\text { Causas }\end{array}$ & Total \\
\hline Perinatais & 92 & 3 & 3 & 2 & 1 & 6 & 107 \\
\hline Congênitas & 1 & 15 & 1 & 二 & - & 2 & 19 \\
\hline Diarréia & 1 & 2 & 8 & 1 & 8 & 1 & 21 \\
\hline Pneumonia & 4 & - & 1 & 11 & 3 & 1 & 20 \\
\hline Desnutriçāo & - & - & 2 & 1 & 6 & 1 & 10 \\
\hline Outras Causas & 1 & 1 & 1 & 2 & 一 & 13 & 18 \\
\hline$\overline{\text { Total }}$ & 99 & 21 & 16 & 17 & 18 & 24 & 195 \\
\hline
\end{tabular}

* DEAN, A.G. et al. Epi Info, version 5. USD, Incorporated, Stone Mountain, Georgia, 1990.
**GUSTAFSON, T.L. True Epistat. Epistat Services, Texas, 1987. 
Tabela 2 - Concordância observada (CO) entre as Declarações de Óbito (DO) originais e as obtidas após revisão (modificada) e respectivos coeficientes Kappa, região metropolitana de Belo Horizonte, 1989).

\begin{tabular}{|c|c|c|c|c|c|c|c|}
\hline \multirow{3}{*}{$\begin{array}{l}\text { Grupo } \\
\text { de } \\
\text { causa }\end{array}$} & \multicolumn{4}{|c|}{ DO } & \multirow{3}{*}{$\mathrm{CO}$} & \multirow{3}{*}{$\begin{array}{c}\text { Kappa } \\
\text { (IC 95\%) }\end{array}$} & \multirow{3}{*}{ Relação* } \\
\hline & \multicolumn{4}{|c|}{ Original Modificada } & & & \\
\hline & № & $\%$ & № & $\%$ & & & \\
\hline Perinatais & 107 & 54,9 & 99 & 50,8 & 0,89 & $0,77(0,63-0,91)$ & 0,93 \\
\hline Congênitas & 19 & 9,7 & 21 & 10,8 & 0,95 & $0,72(0,58-0,86)$ & 1,11 \\
\hline Diarréia & 21 & 10,8 & 16 & 8,2 & 0,89 & $0,37(0,24-0,51)$ & 0,76 \\
\hline Pneumonia & 20 & 10,3 & 17 & 8,7 & 0,92 & $0,55(0,41-0,69)$ & 0,85 \\
\hline Desnutrição & 10 & 5,1 & 18 & 9,2 & 0,92 & $0,39(0,25 \cdot 0,52)$ & 1,80 \\
\hline Outras & 18 & 9,2 & 24 & 12,3 & 0,92 & $0,57(0,44 \cdot 0,71)$ & 1,33 \\
\hline Total & 195 & 100 & 195 & 100 & 0,74 & $0,62(0,55-0,69)$ & 1,00 \\
\hline
\end{tabular}

* Relação = DO Modificada / DO original

A prematuridade (categoria 765) como causa básica foi pouco significativa mesmo na DO original, já que as regras de seleção da causa básica especificam que esta não deve ser selecionada se constar na DO qualquer outra causa de mortalidade neonatal. Entretanto, a contribuição desta afecção para a mortalidade foi evidente, sendo citada em $60 \%$ dos óbitos neonatais (como causa básica ou associada).

Em relação à mortalidade pós-neonatal, os óbitos da DO original estavam mais uniformemente distribuídos entre os 6 grupos de causa, sendo que as diarréias, pneumonias e desnutrição, de menor índice de concordância, tiveram relativamente maior peso que as causas perinatais e as anomalias congenitas. Ao se estudar a associação entre a desnutrição e outras causas de óbito, verificou-se que a desnutrição foi citada em $76,9 \%$ dos óbitos em que a diarréia foi selecionada como causa básica nos atestados modificados. Em relação à pneumonia, a desnutrição esteve associada $18,7 \%$ das vezes, sendo que para as demais causas o percentual foi de $21,6 \%$ (Tabela 4 ).

Observou-se grande intercâmbio entre a desnutrição, diarréia e pneumonia quando da seleção da causa básica "verdadeira" (atestado modificado). Isto ficou patente quando se consideraram os óbitos pós-neonatais por diarréia, pneumonia e desnutrição como um conjunto: de 43 óbitos devidos a uma dessas causas na DO original, somente 5 deles $(11,6 \%)$ tiveram outras causas básicas selecionadas após a investigação.

\section{Discussão}

Apesar da morte representar a última etapa do processo saúde-doença, as estatísticas de mortalidade entre nós são ainda muito importantes para avaliação do estado de saúde da população, pois freqüentemente são as únicas disponíveis em base populacional. Muitos médicos desconhecem entretanto a importância do preenchimento correto dos atestados de óbito para as estatísticas de mortalidade, considerando-o apenas como uma atividade burocrática para o sepultamento ${ }^{8}$.

Nas últimas décadas, as escolas médicas no Brasil buscaram transmitir para seus alunos uma visão mais ampla do trabalho do médico, incluindo suas responsabilidades nas estatísticas de saúde. Apesar disto, as estatísticas de mortalidade, principalmente os dados relativos às causas de óbito, continuam recebendo muitas críticas ${ }^{2,9,12}$.

No presente trabalho verificou-se que, ao se fazer a comparação dos resultados individuais relativos à causa básica dos atestados originais e a selecionada pela equipe de investigação, o índice de concordância geral foi baixo $(74,4 \%)$, ou seja, $25,6 \%$ dos atestados foram considerados incorretos em relação à declaração da verdadeira causa básica do óbito. $\mathrm{O}$ índice kappa foi de 0,62 , sendo de 0,61 para os óbitos neonatais e de somente 0,47 para os óbitos pósneonatais. Estes resultados foram quase idênticos aos obtidos em estudo semelhante realizado na região metropolitana do Rio de Janeiro, em $1986^{2}$, mostrando que a pouca confiabilidade da declaração da causa básica das mortes infantis segue padrão semelhante em regiões metropolitanas do Brasil.

A maior concordância encontrada no grupo dos óbitos neonatais pode ser explicada pelo grande número de óbitos ligados à prematuridade, quer devido a patologias que atuaram como causa, quer devido às complicações desta condição. Além disso, o grupo das afecções perinatais, responsável pela maioria dos óbitos analisados, é bastante amplo, levando a que, mesmo mudando de categoria após a

Tabela 3 - Número e percentagem de óbitos infantis, segundo grupo etário e concordância entre atestados originais e modificados com respectivos coeficientes Kappa, região metropolitana de Belo Horizonte, 1989.

\begin{tabular}{lcccccc}
\hline Grupo & \multicolumn{2}{l}{ Concordantes } & \multicolumn{2}{l}{ Discordantes } & Total (No) & Kappa (IC 95\%) \\
etário & № & $\%$ & NNo & $\%$ & & \\
Neonatais & 98 & 88,3 & 13 & 11,7 & 111 & $0,61(0,49-0,74)$ \\
Pós-neonatais & 47 & 56,0 & 37 & 44,0 & 84 & $0,47(0,38-0,57)$ \\
\hline Total & 145 & 74,4 & 50 & 25,6 & 195 & $0,62(0,55-0,69)$ \\
\hline
\end{tabular}


Tabela 4 - Número de óbitos pós-neonatais segundo causas básicas de óbito e número e percentagem de óbitos com desnutrição como causa associada, região metropolitana de Belo Horizonte, 1989.

\begin{tabular}{lccc}
\hline Causa básica & Total de óbitos & \multicolumn{2}{c}{$\begin{array}{c}\text { Desnutrição como } \\
\text { causa associada } \\
\text { No }\end{array}$} \\
Desnutriçăo & 18 & - & - \\
Pneumonia & 16 & 3 & 18,7 \\
Diarréia & 13 & 10 & 76,9 \\
Demais causas & 37 & 8 & 21,6 \\
\hline Total & 84 & 21 & 25,0 \\
\hline
\end{tabular}

investigação, a causa básica continuava sendo classificada neste capítulo. Deve ser considerado ainda que os prontuários hospitalares para esta faixa etária estavam freqüentemente incompletos, o que favoreceu a concordância, já que os óbitos tenderam a ser classificados de forma mais genérica.

A menor confiabilidade da DO para os óbitos pósneonatais, devidos principalmente à desnutrição, diarréias e pneumonias, pode ser relacionada à maior dificuldade de definição das causas básicas e contribuintes nestes casos, onde tem peso acentuado a relação sinérgica infeç̧ão-desnutrição. Para estas patologias há dificuldade em seguir o princípio da causa básica da morte, principalmente pelo fato de que a etiologia e a sequiência de desenvolvimento das doenças não podem ser determinadas em muitos casos. Existe então certo grau de subjetividade na definição da causa básica e das causas associadas, já que fica a critério do profissional definir as causas mais relevantes do processo mórbido que devem constar nas declarações de óbito ${ }^{2}$.

Além da questão relativa ao correto preenchimento da DO, pelos médicos, chamou atenção no presente estudo o problema relativo à codificação da causa básica do óbito. Os atestados médicos modificados foram preenchidos pela equipe de pesquisa, segundo as regras de preenchimento da DO, citando-se

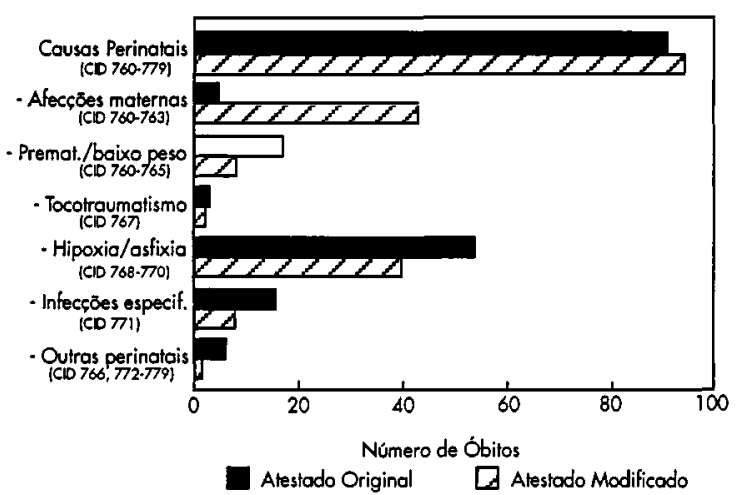

Figura. Óbitos neonatais por afecções perinatais, segundo causas básicas de morte no atestado original e no atestado modificado, região metropolitana de Belo Horizonte - 1989. na parte I, por último, a patologia causa básica do obito. Entretanto, quando enviados para codificação, vários atestados não tiveram esta última patologia selecionada como causa básica. Foi feita então revisão dos atestados discordantes, sendo discutida com a coordenação da equipe de codificação os motivos de discordância. Verificou-se que a desnutrição grave só era selecionada como causa básica quando associada à gastrenterite ou diarréia, não o sendo quando o médico citava enteroinfecção ao invés de gastrenterite. Como estas duas patologias são equivalentes do ponto de vista clínico e epidemiológico, a desnutrição grave foi considerada no presente estudo como causa básica do óbito para ambos os casos, levando a que 7 óbitos anteriormente codificados com o CID 009 (diarréia) passassem para o CID 263 (desnutrição).

Outro problema relacionado à codificação foi o relativo a se considerar como código 771.8 todas as septicemias, o que levava a uma sobrenumeração da mortalidade por afecções perinatais, grupamento ao qual pertence o código 771.8, e a uma subestimação dos óbitos infantis por causas infecciosas. Assim, 15 óbitos da amostra estudada devidos à septicemia (código CID 038), correspondendo a um total estimado de 218 óbitos em 1989, estavam "desaparecidos" entre as causas perinatais. A utilização indevida pelos codificadores regionais do código 771.8 (infecções específicas do período perinatal) para os casos de septicemia pós-neonatal explicou o quase "desaparecimento" desta patologia como causa de mortalidade infantil nas estatísticas oficiais publicadas no Estado, a partir de 1984. Logo no início da pesquisa este erro foi detectado e corrigido. Vale lembrar, entretanto, que esta simples alteração nas normas de codificação resultou em um aumento artificial da mortalidade por causas perinatais nas estatísticas divulgadas no Estado.

Apesar do presente estudo não ter como objetivo a validação das práticas de seleção da causa básica, chamou atenção a possibilidade de erros devidos a isto. Sabe-se que estudos que investigam validade da certificação médica da causa de óbito e práticas de codificação ao mesmo tempo são importantes, pois algumas diferenças na certificação podem ou não ser compensadas no processo de codificação ${ }^{4}$, sendo que diferenças na certificação e codificação das causas de óbito podem ser determinantes importantes de diferenças no perfil da mortalidade.

O alto percentual de discordância encontrado no presente estudo pode se tornar ainda mais preocupante ao se considerar que se refere à discordância entre grupos de causas, pois não foi avaliada a discrepância da causa revisada com a causa registrada no atestado quando as mesmas, apesar de pertencerem ao mesmo grupo, eram classificadas em categorias diferentes. Por exemplo, ao considerar 14 categorias de causas infecciosas e deficiências nutricionais para análise da mortalidade de crianças entre 7 e 364 dias de idade, 
outros autores encontraram uma discordância bem maior $(72,1 \%)^{12}$.

Este dado, alarmante se considerado do ponto de vista de cada óbito individual, passa a ter menos importância quando se verifica que, em nível das estatísticas de mortalidade, a alteração na composição dos principais grupos de causa foi pequena, por compensação interna das discordâncias. Verificou-se portanto que, de modo geral, não houve mudança significativa na composição da mortalidade infantil pelos cinco principais grupos de causa após a investigação. As causas perinatais permaneceram como primeira causa de mortalidade infantil após o preenchimento do "novo atestado". Houve modificação na proporção relativa das pneumonias e anomalias congênitas como causas de óbito, passando estas para 10,8\% do total e aquelas para $8,7 \%$, após a investigação. A maior alteração entretanto, ficou com a desnutrição, responsável por $9,2 \%$ dos óbitos infantis após a investigação, ou seja, quase o dobro do número inicial. Além disso, tanto a prematuridade quanto a desnutrição, considerados fatores importantes de vulnerabilidade à morte ${ }^{14}$, estavam presentes em mais da metade dos óbitos neonatais e pós-neonatais como causa básica ou associada.

A abordagem da mortalidade infantil pelo enfoque de causas múltiplas permitiria avaliar a importância de todas as causas ou estados patológicos que levaram à morte, ajudando no conhecimento dos acontecimentos que resultaram no óbito's. Isto se torna particularmente importante em países subdesenvolvidos como o Brasil, onde a interação doença infecciosa-desnutrição, já amplamente conhecida ${ }^{15}, 16$, ainda é responsável pela perda precoce de muitas vidas.

Assim, mesmo que a validade da declaração da causa básica dos atestados individuais possa ser questionada, as estatísticas agregadas desses óbitos fornecem indicação suficiente dos principais grupos de doenças para serem usadas na investigação de problemas de saúde pública.

\section{Agradecimentos}

À Helen Maria Ramos de Oliveira, pela colaboração dada na revisão final do artigo, a Henrique Leonardo Guerra, pela ajuda na utilização do programa Epistat, a Mark Drew Crosland Guimarães, pelas úteis sugestões, e à equipe do Centro de Estatística e Informação da Fundação João Pinheiro, pela codificação dos óbitos.

MENDONÇA,E.F.etal. [Realiability of the medicalcertificates of underlying cause of infant deaths in a metropolitan region of southeastern Brazil]. Rev. Saúde Pública, 28:385-91, 1994. The quality of official information on underlying causes of infant deaths was studied on the basis of data collected for a popullation-based study of the surveillance of infant mortality in the metropolitan region of Belo Horizonte, Brazil in 1989. The survey included the analysis of a sample of infant deaths carried out by comparing the underlying causes of death as coded on death certificates to those recorded by a group of doctors who abstracted information from hospital records. We verified that $11.7 \%$ of neonatal deaths did not have the underlying cause of death confirmed by the investigation (kappa $=0.61$ ), and neither did $44.0 \%$ of post-neonatal deaths (kappa $=0.47$ ). It is believed that this major disagreement among post-neonatal deaths is due to the close correlation observed among the major causes of death within this group (pneumonia, diarrhoea and malnutrition). For example, associated malnutrition was observed in $76.9 \%$ of those cases in which diarrhoea was coded as the underlying cause of death. It was concluded that the quality of the death certificates is not satisfactory. However, the composition of the main groups of causes presented no significant alteration after investigation and may be used in public health surveillance, especially if we regard pneumonia, diarrhoea and malnutrition as a group with the same determinants. Unfortunately, this group still accounts for a great number of otherwise avoidable deaths in Brazil.

Keywords: Underlying cause of death. Infant mortality. Death certificates.

\section{Referências Bibliográficas}

1.BARROS,F.C.; VICTORA,C.G.;TEIXEIRA, A.M.B.; PUERTO FILHO, M. Mortalidade perinatal e infantil em Pelotas, Rio Grande do Sul: nossas estatísticas são confiáveis? Cad. Saúde Pública, 1:348-58, 1985.

2. CARVALHO, M.L.; NIOBEY, F.M.L.; MIRANDA, N.N.; SABROZA, P.C. Concordância na determinação da causa básica de óbito em menores de um ano na região metropolitana do Rio de Janeiro, 1986. Rev. Saúde Pública, 24:207, 1990.

3. The DEATH certificate-fact or fiction? S. Afr. Med. J., 69:4023, 1986.

4. ERHARDT, C.L. What is "the cause of death"? JAMA, 168:161$8,1958$.

5. FLEISS, J.L. Statistical methods for rates and proportions. New York, John Wiley and Sons, 1981.

6. FONSECA, L.A.M.\& LAURENTI, R. A qualidade da certificação médica da causa de morte em São Paulo, Brasil. Rev. Saúde Pública, 8:21-9, 1974.

7. HISCH, C.S. Determining causes and mechanisms of death: death certification. apud. Kircher, T. \& Anderson, R. E. Cause of death; proper completion of the death certificate, JAMA, 258: 349-52, 1987.

8. LAURENTI, R.; MELLO JORGE, M.H.P.; LEBRĀO, M.L.; GOTLIEB, S.L.D. Estatísticas de saúde. São Paulo, EPU/ EDUSP, 1985.

9. LAURENTI, R. \& MELLO JORGE, M.H.P. Oatestado de óbito. São Paulo, Centro da OMS para a Classificação de Doenças em Português, 1983.

10. MILANESI, M.L. \& LAURENTI, R. O estudo interamericano 
de mortalidade em São Paulo. I - Estado atual da certificação médica da causa de óbito no distrito da Capital. Rev. Assoc. Méd. Bras., 10:111-6, 1964.

11. MONTEIRO, C.A. Critérios antropométricos no diagnóstico de desnutrição em programas de assistência à saúde. Rev. Saúde Pública, 18:209-17, 1984

12.NOBRE, L.C.; VICTORA, C.G.;BARROS, F.C;LOMBARDI, C.; TEIXEIRA, A.M.B.; FUCHS, S.C. Avaliação da qualidade da informação sobre a causa básica de óbitos infantis no Rio Grande do Sul (Brasil). Rev. Saúde Pública, 23:207$13,1989$.

13. ORGANIZAÇÃO MUNDIAL DA SAÚDE. Manual de classificação estatística internacional de doenças, lesões e causas de óbitos, $9^{\mathrm{a}}$ rev. 1975. São Paulo, Centro da OMS para Classificação de Doenças em Português, 1978.

14. ORGANIZAÇÃO PANAMERICANA DE LA SALUD. Las condiciones de salud en las Americas. Washington, DC, 1990. (OPAS - Publicación Cientifica, 524).
15. PUFFER, R.R. Nuevos enfoques para los estudios epidemiologicos sobre estadísticas de mortalidad. Bol. Ofic. Sanit. Panam., 107:277-94, 1989.

16. SAAD, P.M. Mortalidade infantil por causas no Estado de São Paulo (Brasil), em 1983: análise sob perspectiva de causas múltiplas de morte. Rev. Saúde Pública, 20:481-8, 1986.

17. VIEGAS, J.M.S. \& DOLABELA, R.F. Estudo qualitativo das estatísticas de nascimentos e óbitos: o sub-registro em Minas Gerais e região metropolitana de Belo Horizonte: resultados definitivos do Censo Demográfico de 1980. Indic. Conjunt. Belo Horizonte, 8:485-95, 1986.

Recebido para publicaçāo em 8. 2. 1994 Reapresentado em 11.8.1994 Aprovado para publicação em 13. 9. 1994 